Original Article

\title{
Micro-scale habitat associations of woody plants in a neotropical cloud forest
}

\author{
1. Alicia Ledo ${ }^{1, *}$, \\ 2. David F.R.P. Burslem², \\ 3. Sonia Condés ${ }^{1}$, \\ 4. Fernando Montes ${ }^{3}$
}

Article first published online: 20 DEC 2012

DOI: $10.1111 /$ jvs.12023

(c) 2012 International Association for Vegetation Science

Issue

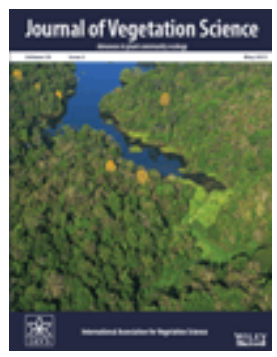

\section{Journal of Vegetation Science}

Early View (Online Version of Record published before inclusion in an issue) (/journal/10.1111/(ISSN)1654-1103 learlyview)

Additional Information

\section{How to Cite}

Ledo, A., Burslem, D. F.R.P., Condés, S., Montes, F. (2012), Micro-scale habitat associations of woody plants in a neotropical cloud forest. Journal of Vegetation Science. doi: 10.1111/jvs.12023

\section{Author Information}

1 Universidad Politécnica de Madrid, Escuela Técnica Superior de Ingenieros de Montes, Ciudad Universitaria, Madrid, Spain

2 School of Biological Sciences, University of Aberdeen, Aberdeen, AB24 3UU, UK

3 CIFOR-INIA, Madrid, Spain 
(fmontes@inia.es)

*corresponding author, alicialedo@gmail.com (mailto:alicialedo@gmail.com)

\section{Publication History}

1. Article first published online: 20 DEC 2012

2. Manuscript Accepted: 22 OCT 2012

3. Manuscript Received: 26 JAN 2012

\section{Funded by}

- Universidad Politécnica de Madrid

- Consejo Social de la Universidad Politécnica de Madrid

- Abstract

- Article (/doi/10.1111/jvs.12023/full)

- References (/doi/10.1111/jvs.12023/references)

- Supporting Information (/doi/10.1111/jvs.12023/suppinfo)

- Cited By (/doi/10.1111/jvs.12023/citedby)

View Full Article with Supporting Information (HTML) (/doi/10.1111/jvs.12023/full) Get PDF (398K) (/doi/10.1111/jvs.12023/pdf)

\section{Keywords:}

Andes; Dispersal limitation; Habitat partitioning; Montane tropical forest; Peru; Spatial pattern; Species co-e

\section{Abstract \\ Questions}

Species-habitat associations may contribute to the maintenance of species richness in tropical forests, but previous research has been conducted almost exclusively in lowland forests and has emphasized the importance of topography and edaphic conditions. Is the distribution of woody plant species in a Peruvian cloud forest determined by microhabitat conditions? What is the role of environmental characteristics and forest structure in habitat partitioning in a tropical cloud forest?

\section{Location}

Cloud Forest, north Peruvian Andes.

\section{Methods}

We examined species-habitat associations in three 1-ha plots using the torus-translation method. We used three different criteria to define habitats for habitat partitioning analyses, based on microtopography, forest structure and both sets of factors. The number of species associated either positively or negatively with each habitat was assessed.

\section{Results}


Habitats defined on the basis of environmental conditions and forest structure discriminated a greater number of positive and negative associations at the scale of our analyses in a tropical cloud forest.

\section{Conclusions}

Both topographic conditions and forest structure contribute to small-scale microhabitat partitioning of woody plant species in a Peruvian tropical cloud forest. Nevertheless, canopy species were most correlated with the distribution of environmental variables, while understorey species displayed associations with forest structure.

View Full Article with Supporting Information (HTML) (/doi/10.1111/jvs.12023/full) Get PDF (398K) (/doi/10.1111/jvs.12023/pdf)

\section{More content like this}

Find more content:

- like this article (/advanced/search/results?articleDoi=10.1111/jvs.12023\&scope=allContent\&start=1\& $\underline{\text { resultsPerPage }=20)}$

Find more content written by:

- Alicia Ledo (/advanced/search/results?searchRowCriteria[0].queryString="Alicia Ledo"\&searchRowCriteria[0].fieldName=author\&start=1\&resultsPerPage=20)

- David F.R.P. Burslem (/advanced/search/results?searchRowCriteria[0].queryString="David F.R.P. Burslem"\&searchRowCriteria[0].fieldName=author\&start=1\&resultsPerPage=20)

- Sonia Condés (/advanced/search/results?searchRowCriteria[0].queryString="Sonia Cond\%C3 \%A9s"\&searchRowCriteria[0].fieldName=author\&start=1\&resultsPerPage=20)

- Fernando Montes (/advanced/search/results?searchRowCriteria[0].queryString="Fernando Montes"\&searchRowCriteria[0].fieldName=author\&start=1\&resultsPerPage=20)

- All Authors (/advanced/search/results?searchRowCriteria[0].queryString="Alicia Ledo" "David F.R.P. Burslem" "Sonia Cond\%C3\%A9s" "Fernando Montes"\&searchRowCriteria[0].fieldName=author\&start=1\& resultsPerPage $=20$ ) 